LUNG TRANSPLANTATION

\title{
Combined donor specific transfusion and anti- CD154 therapy achieves airway allograft tolerance
}

\author{
W Chalermskulrat, K P McKinnon, W J Brickey, I P Neuringer, R C Park, D G Sterka, \\ B R Long, P McNeillie, R J Noelle, J P Ting, R M Aris
}

Thorax 2006;61:61-67. doi: 10.1136/thx.2005.047316

See end of article for authors' affiliations

Correspondence to:

Dr R M Aris, Division of Pulmonary Diseases and Critical Care Medicine, CB \#7020, Bioinformatics Building, Room 4131, Chapel Hill, NC 27599 ,

USA; aris@med.unc.edu

Received 27 May 2005 Accepted 18 October 2005 Published Online First 27 October 2005

\begin{abstract}
Background: The state of tolerance allows long term graft survival without immunosuppressants. Lung transplantation tolerance has not been consistently achieved in either small or large animal models.

Methods: The mechanisms and effectiveness of a tolerance induction protocol consisting of donor specific transfusion (DST; day 0) and a short course of co-stimulatory blockade (anti-CD154 antibody; days -7 , $-4,0$ and +4 ) were studied in the mouse heterotopic tracheal transplant model of chronic lung rejection. C57BL/6 mice received BALB/c tracheal grafts (day 0) and were treated with DST alone, anti-CD154 alone, the combination (DST/anti-CD154), or no treatment. No non-specific immunosuppressants were used.

Results: DST/anti-CD154 in combination, but neither treatment alone, markedly prolonged the lumen patency and survival ( $>100$ days) of fully histo-incompatible allografts ( $p<0.05$ versus control allografts at every time point studied up to 16 weeks) without immunosuppression. This protocol was donor antigen specific as third party grafts $(\mathrm{C} 3 \mathrm{H})$ were promptly rejected. In addition, DST/anti-CD154 did not result in mixed chimerism but induced transplantation tolerance via a peripheral mechanism(s), which included significantly reduced cytotoxic $T$ cell activity $(p<0.001)$ and a significantly increased percentage of CD4+CD25+ cells $(p=0.03)$.

Conclusions: The DST/anti-CD154 protocol successfully induced and maintained long term, donor specific tolerance in the mouse heterotopic airway graft model of chronic lung rejection. This finding may lead us closer to successful tolerance induction in lung transplantation.
\end{abstract}

L ung transplantation has become an established therapeutic option for patients with end stage pulmonary disease. Its success is markedly limited by chronic lung rejection or obliterative bronchiolitis (OB), manifested clinically as progressive airway obstruction. Obliterative bronchiolitis is the leading cause of late death after lung transplantation and is the principal reason that the 5 and 10 year survival rates (approximately $45 \%$ and $20 \%$, respectively) are $20-30 \%$ lower, on an absolute basis, than those after heart, kidney, or liver transplantation. Currently available immunosuppressants, which must be maintained for life, are neither transplant antigen specific nor effective in treating or preventing chronic graft rejection. In addition, they possess many serious adverse effects. A more attractive method of inducing graft acceptance is through selectively coaxing the recipient's immune response into accepting the transplanted organ as self, while maintaining normal immune reactivity against non-graft foreign antigens. This approach, called tolerance induction, has become the ultimate goal of transplant immunology. Tolerance research in the arena of lung transplantation has been very limited and has lagged behind that in other organs. In fact, tolerance of lung allografts has not been consistently achieved in either small or large animal models. ${ }^{1}$

While allorecognition, through engagement of the $\mathrm{R}$ cell receptor (TCR) with the donor derived MHC peptide complex, is the mandatory event for allospecific $\mathrm{T}$ cells to recognise donor antigens (or signal 1), it is the presence or absence of the "co-stimulation signal" (or signal 2) that educates allospecific $\mathrm{T}$ cells to either become fully activated, proliferate, and develop into effector cells, or to become anergic or tolerogenic, respectively. ${ }^{2}$ The two most extensively studied co-stimulatory pathways are the B7/CD28 and CD40/CD154
(CD40L). ${ }^{3}$ Through competitive binding to B7 molecules on antigen presenting cells (APCs), CTLA-4 Ig (a soluble form of the high affinity receptor to $\mathrm{B} 7$ molecules) blocks CD28 signalling to the $\mathrm{T}$ cells, therefore limiting the activation of the alloreactive $\mathrm{T}$ cells. Treatment with CTLA4-Ig alone supports long term allograft survival of islet cells ${ }^{4}$ and hearts in animal models. ${ }^{5}$ However, since a CD28 independent CTLA4 signal might deliver a strong negative signal to CD4+ T cells and thus interrupt the CTLA4/B7 interaction, alloreactive $\mathrm{T}$ cells are not effectively shut down. ${ }^{6}$ Other means by which to disrupt $\mathrm{T}$ cell activation includes blockade of the CD40/CD154 co-stimulatory pathway which, in turn, limits the maturation of APCs and down-modulates the B7/CD28 interaction. ${ }^{7}$ In the mouse model of chronic lung rejection, the greater importance of CD40/CD154 compared with B7/ CD28 has been demonstrated by the finding that recipient mice deficient in CD154 (but not CD28) expression on T cells reject tracheal allografts more slowly than wild-type recipient mice. $^{8}$

Translating tolerance protocols from one organ to another has been elusive. Thus, the success of a tolerance induction protocol in one organ may not necessarily ensure the success in others. ${ }^{1}$ We hypothesise that selective blockade of immune responses that allows allorecognition of donor antigen by allospecific T lymphocytes through specific TCRs (signal 1), but prevents co-stimulatory signalling (signal 2), may prevent the initiation of rejection in an antigen specific manner. One tolerance induction protocol-namely,

Abbreviations: APCs, antigen presenting cells; CFSE, carboxyfluorescein diacetate succinimidyl ester; CTL, cytotoxic lymphocyte; DST, donor specific transfusion; $M H C$, major histocompatibility complex; $O B$, obliterative bronchiolitis; TCR, T cell receptor; $T_{R}$, regulatory $T$ cells 
combined donor specific transfusion (DST) and anti-CD154 monoclonal antibody (DST/anti-CD154) - has emerged as a robust tolerance induction protocol in non-lung transplant models including islet, ${ }^{9}$ heart, ${ }^{10}$ and skin $^{11}$ allografts. Its effectiveness in lung transplantation has not been tested. Using the mouse model of chronic lung rejection (or obliterative bronchiolitis) which most faithfully models chronic airway rejection, ${ }^{12}{ }^{13}$ we tested the hypothesis that DST/anti-CD154 therapy induces lung transplantation tolerance and explored the immunological mechanisms of action.

\section{METHODS}

Mice

Female BALB/c $\left(\mathrm{H}-2^{\mathrm{d}}\right)$ or third-party $\mathrm{C} 3 \mathrm{H}\left(\mathrm{H}-2^{\mathrm{k}}\right)$ donors and C57BL/6 $\left(\mathrm{H}-2^{\mathrm{b}}\right)$ recipient mice (8-12 weeks old) were used (Harlan Labs; Indianapolis, IN, USA). Mice were maintained following the guidelines of the National Institutes of Health Guide for the Care and Use of Laboratory Animals under an approved IACUC protocol at the University of North CarolinaChapel Hill.

\section{Transplantation}

Heterotopic tracheal transplantation surgery, tissue processing and staining, image acquisition, and the measurement of lumen fibroproliferation, the determinant of airway rejection, were performed as previously described. ${ }^{14-17}$

\section{Treatment protocols}

DST consisted of a single dose of $10^{7}$ freshly isolated donor splenocytes given to recipients on day -7 relative to transplantation. ${ }^{18}$ Briefly, single cell suspensions were obtained by passing splenocytes through a $70 \mu$ m nylon filter (BD Biosciences, San Jose, CA, USA), subjected to hypotonic erythrocyte lysis, counted, diluted in $200 \mu \mathrm{l}$ phosphate buffered saline (PBS), and transfused into the tail vein of recipient mice. The hamster anti-mouse CD154 monoclonal antibody (MR-1, Bioexpress, West Lebanon, NH, USA) was administered intraperitoneally at a dose of $0.5 \mathrm{mg} / \mathrm{mouse}$ on days $-7,-4,0$, and +4 . Recipient mice received either of the treatments, or both combined, or none (fig 1). No

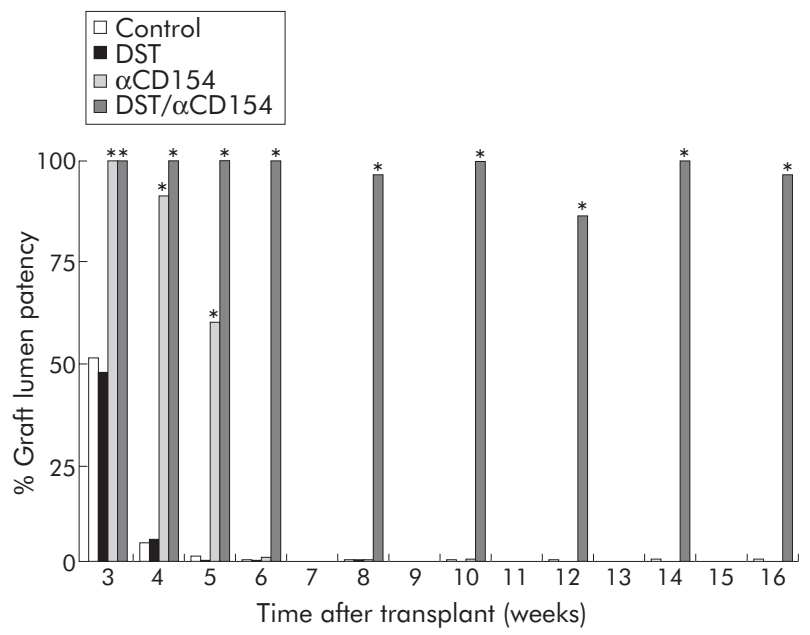

Figure 1 DST/anti-CD154 induces and maintains long term airway allograft tolerance. Without the use of immunosuppressants, treatment with DST/ $\alpha C D 154$, but not either DST or anti-CD154 alone, maintained lumen patency and prolonged tracheal allograft survival indefinitely (>100 days). The freedom from airway rejection in allografts transplanted into recipients under treatments of DST, anti-CD154, DST/ anti-CD154, or no treatment is shown as the mean percentage of airway lumen patency $(n=5-11$ and SDs $\leqslant 18 \%$ for each treatment arm at each time point). ${ }^{*} p<0.05$ compared with control allografts at the same time point. immunosuppressants were used. Tolerance was defined as survival for $>100$ days as previously described. ${ }^{18} 19$

\section{Cytotoxic lymphocyte (CTL) assay}

As previously described, ${ }^{20}$ splenocytes from transplanted or naïve C57BL/6 mice were restimulated with gamma irradiated (20 Gy) donor (BALB/c) or control syngeneic (C57BL/ 6) stimulator splenocytes at a $1: 1$ ratio for 6 days before testing their efficacy as effector cells. At $37^{\circ} \mathrm{C}$ in $5 \% \mathrm{CO}_{2}$, effector cells were then co-cultured in triplicates for 4 hours with 5000 concanavalin-A stimulated $\left[{ }^{3} \mathrm{H}\right]$-thymidine labelled target (allogeneic or self) blast cells with effector to target cell ratios ranging from 100:1 to $12.5: 1$ in 96 well plates. The cells were harvested and the specific $\left[{ }^{3} \mathrm{H}\right]$ release was determined using liquid scintillation counting on a Microtiter Plate Reader (Packard Instrument, Meriden, CT, USA). Percentage cytotoxicity was calculated as the difference in spontaneous lysis and targeted release over spontaneous lysis.

\section{Carboxy-fluorescein diacetate succinimidyl ester (CFSE) staining}

As previously described, ${ }^{20}$ single cell suspensions of freshly isolated splenocytes $\left(10^{7} / \mathrm{ml}\right.$ in PBS) were incubated with an equal volume of $10 \mu \mathrm{M}$ CFSE (Molecular Probes, Eugene, OR, USA) in the dark with periodic agitation $\left(37^{\circ} \mathrm{C}, 10 \mathrm{~min}\right)$. Unbound CFSE was quenched with an equal volume of fetal bovine serum (Sigma-Aldrich, St Louis, MO, USA). In order to determine the fate of transfused donor splenocytes in vivo, CFSE labelled cells were washed three times and transfused into the tail vein of recipient mice. After 12 hours the fluorescence intensities of single cell suspensions obtained from the recipient's spleen, axillary lymph nodes, and the thymi were analysed by flow cytometry using a FACScan (Becton Dickinson, San Jose, CA, USA) and analysed with FlowJo software (Tree Star, San Carlos, CA, USA).

\section{Immunohistochemistry}

Whole spleen, lymph node, and thymus were embedded in Tissue-Tek OTC (Sakura Finetek, Torrance, CA, USA) and stored at $-20^{\circ} \mathrm{C}$ until use. Frozen sections were air dried, fixed in chilled acetone, and blocked with 5\% normal goat serum (Jackson ImmunoResearch, West Grove, PA, USA). The sections were incubated with HRP conjugated rabbit monoclonal anti-FITC antibody (DakoCytomation, Carpinteria, CA, USA). Optimal dilutions and incubation periods were determined empirically. Duplicate sections on the same slide were incubated with rabbit IgG (Jackson ImmunoResearch) as a negative isotype control. Diaminobenzidine (DAB) (Sigma-Aldrich) was used as the substrate and the sections were counterstained with Light Green (Fisher Scientific Company, Pittsburgh, PA, USA).

\section{Immunofluorocytochemistry for regulatory T cells}

Whole spleen and lymph nodes were incubated with RPMI medium containing $10 \%$ FCS and collagenase IV ( $1 \mathrm{mg} / \mathrm{ml}$; Sigma-Aldrich, St Louis, MO, USA) at $37^{\circ} \mathrm{C}$ for 15 minutes. Single cell suspensions were obtained by passing splenocytes through a $70 \mu \mathrm{m}$ nylon filter (BD Biosciences), subjected to hypotonic erythrocyte lysis, and collected into cold Dulbecco's PBS plus 2\% dialysed serum albumin (Sigma-Aldrich). After blocking with purified rat anti-mouse CD16/32 (III/II FCR, 2.4G2, IgG2b) and $10 \% \mathrm{v} / \mathrm{v}$ mouse IgG $\left(15 \mathrm{~min} ; 4^{\circ} \mathrm{C}\right)$, cells $\left(5 \times 10^{6}\right)$ were incubated on ice with FITC conjugated rat antiCD4 (RM4-5, IgG2a) and PE conjugated rat anti-CD25 (PC61, IgG1) or PE conjugated rat anti-CD45RB (anti-B220, clone RA3-6B2, IgG2a), or appropriate isotype matched Ig controls $\left(30 \mathrm{~min} ; 4^{\circ} \mathrm{C}\right.$ ) (all from BD Pharmingen, San Diego, CA, 


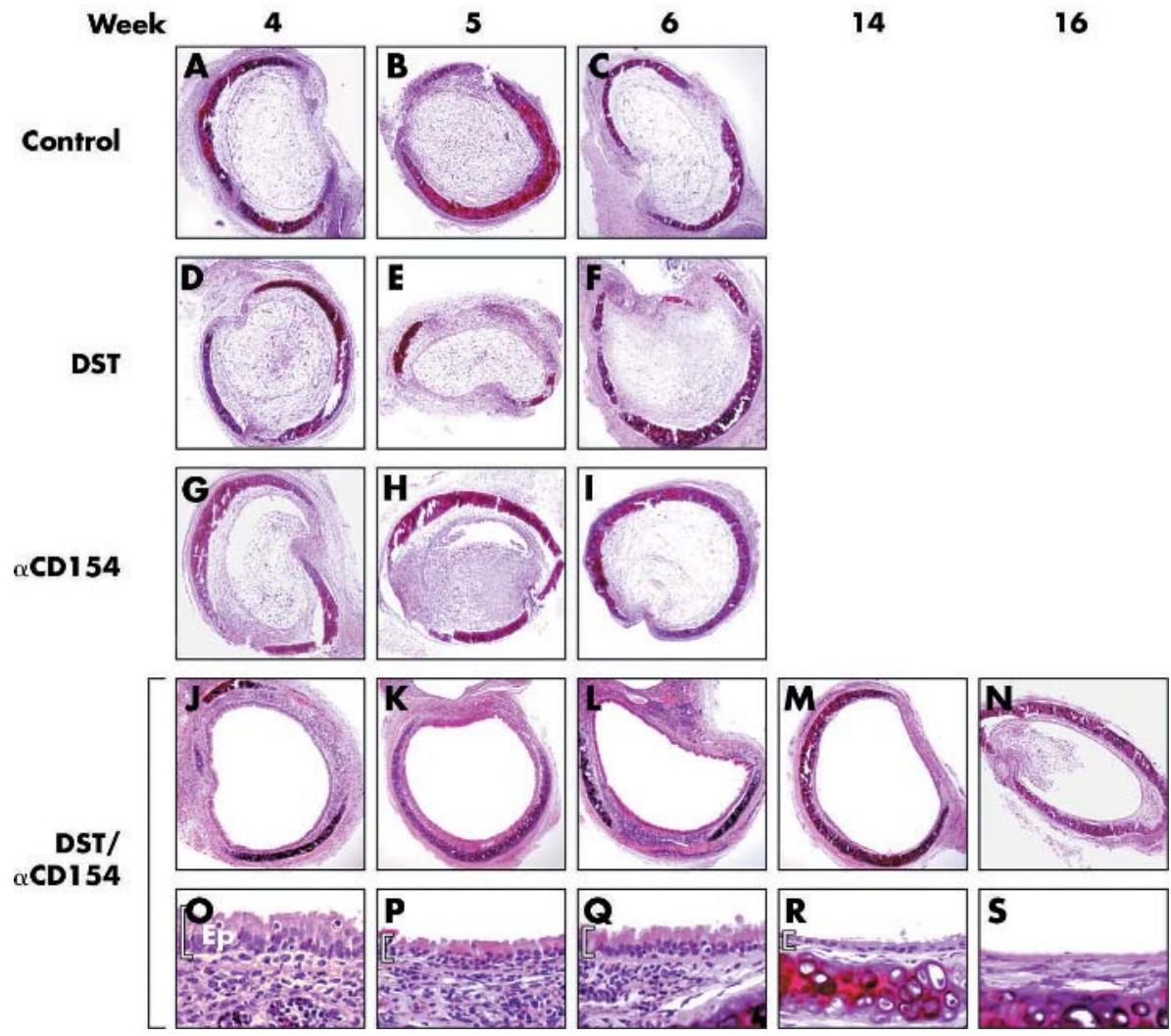

Figure 2 Representative histology of tracheal allografts treated with DST/anti-CD154. Tracheal allografts transplanted into recipients who received DST/anti-CD154 (J-N) displayed a complete or nearly complete patent airway lumen for up to 16 weeks (stain: H\&E, magnification $10 \times$ ). This was not the case for those allografts transplanted into recipients who received DST alone (D-F), anti-CD 154 alone (G-I), or no treatment (A-C). Of note, under DST/anti-CD154 treatment, tracheal allografts maintained epithelialisation for up to 16 weeks and displayed a gradual progressive loss of differentiated epithelium over time $(\mathrm{O}-\mathrm{S}$, magnification $40 \times)$. Ep, epithelium.

USA). Data were acquired on a FACScan and analysed with FlowJo software.

\section{Statistics}

Analyses were performed using the Student's $t$ test with data presented as mean (SD) or, if the data were not normally distributed, non-parametric tests with the data presented as medians with $25-75 \%$ confidence intervals (SigmaStat Software, SPSS Inc, Chicago, IL, USA) as previously described. $^{14-17}$ To test the efficacy of the DST/antiCD154 protocol, different end points in different experimental arms were compared with $t$ tests at each time point and also by analysis of variance (ANOVA) which reflected a comparison across all time points.

\section{RESULTS}

\section{Effect of DST/anti-CD154 on transplantation} tolerance of tracheal allografts

Without treatment, tracheal allografts $(n=6-9$ at each time point) were rapidly rejected resulting in nearly complete airway obliteration at 4 and 5 weeks and complete airway obliteration within 6 weeks (figs 1 and 2A-C). Treatment with DST alone resulted in allograft rejection similar to control allografts $(n=5-8$ at each time point; $p>0.2$ at 3, 4, 5 , and 6 weeks; figs 1 and $2 \mathrm{D}-\mathrm{F}$ ). Treatment with antiCD154 alone modestly prolonged allograft survival up to 5 weeks $(\mathrm{n}=7-8$ at each time point; $\mathrm{p} \leqslant 0.03$ at 3,4 , and 5 weeks). However, the grafts underwent nearly complete lumen obliteration at 6 weeks $(n=6 ; p=0.38)$ and complete lumen obliteration at 8 weeks $(n=7 ; \mathrm{p}=0.40)$, similar to control allografts (figs $\mathrm{l}$ and $2 \mathrm{G}-\mathrm{I}$ ). In sharp contrast, combined treatment with DST/anti-CD154 resulted in markedly preserved allograft lumen patency and prolonged survival ( $>100$ days) $(\mathrm{n}=6-1 \mathrm{l}, \mathrm{p}<0.05 v$ control allografts at each time point from 3 to 16 weeks and $\mathrm{p}<0.001$ using an ANOVA; figs $\mathrm{l}$ and $2 \mathrm{~J}-\mathrm{N}$ ). In addition, with DST/anti-CD154 treatment, tracheal grafts maintained normal epithelialisation for up to 8-10 weeks and cuboidal epithelialisation for up to 14 weeks (fig 2OS), but interestingly had varying amounts of lymphocytic infiltrates.

\section{Effect of DST/anti-D154 on donor specific T lymphocyte cytotoxicity}

The capability of allograft recipients to generate donor specific immune responses was tested by examining CTL activity. Two weeks after transplantation, $\mathrm{T}$ lymphocytes from recipient mice treated with the DST/anti-CD154 combination showed a significant $(\mathrm{p}<0.001)$ decrease in cytotoxicity against donor specific (BALB/c) target cells when compared with those from recipients who received DST alone, anti-CD154 alone, or no treatment (fig 3A). Similar results were observed at 4 weeks after transplantation (data not shown).

\section{Effect of DST/anti-CD154 on donor specific transplantation tolerance}

Third party allografts or third party DST were used to examine the donor specificity of the DST/anti-CD154 protocol. While treatment with BALB/C DST/anti-CD154 in the C57BL/6 recipients markedly prolonged BALB/C tracheal allograft survival, third party $\mathrm{C} 3 \mathrm{H}$ allografts, under BALB/c DST/anti-CD154 treatment, were rejected similar to 

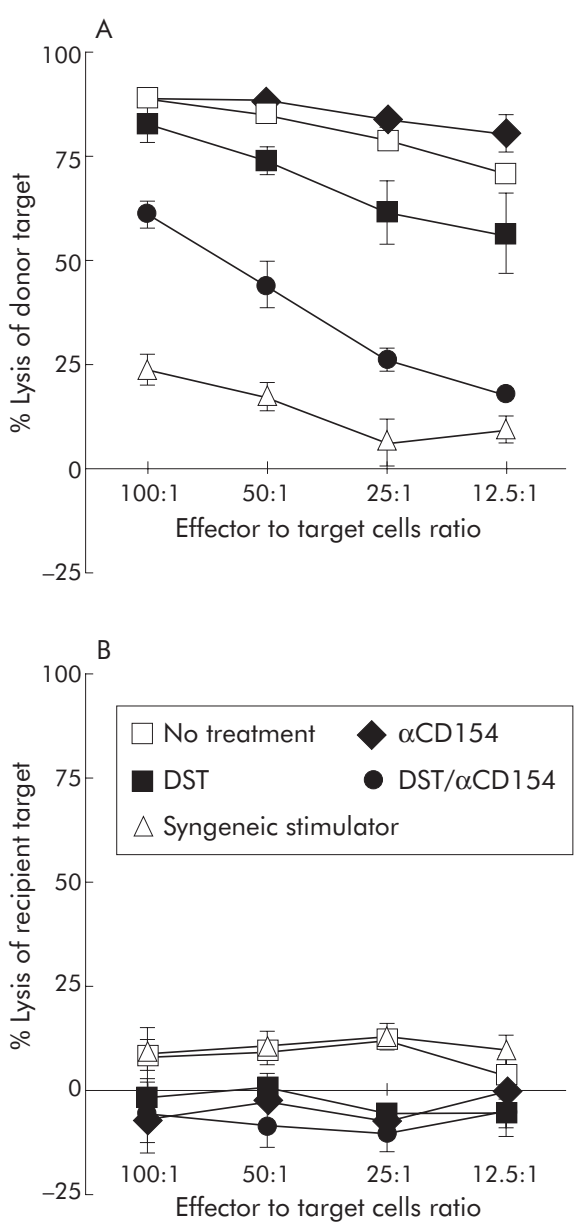

Figure 3 Recipients treated with DST/anti-CD154 exhibited reduced donor specific cytotoxic lymphocytes (CTLs). The secondary CTL assays were performed at 2 weeks after transplantation on splenocytes derived from recipient mice who received different treatments $(n=3$ for each group). Isolated splenocytes were re-stimulated with irradiated spleen cells from mice of the same strain as donor grafts. After 6 days in culture, the re-stimulated recipient cells were harvested and assayed for the ability to lyse radioactive labelled target cells. Under different treatments, CTLs from recipients of $\mathrm{H}^{\mathrm{d}}$ grafts variably lysed allogeneic $\mathrm{H} 2^{\mathrm{d}}$ targets $(\mathrm{A})$, but did not lyse syngeneic $\mathrm{H} 2^{\mathrm{b}}$ control targets (B). The $x$ axis shows effector to target cell ratios. Data are shown as mean (SD) percentage lysis in triplicate cultures from three animals and are representative of two separate experiments.

The SD bars on the no treatment group are too small to be seen on the graph.

$\mathrm{C} 3 \mathrm{H}$ allografts under anti-CD154 treatment alone at 6 weeks $(\mathrm{n}=6$, median patency $15(25-75 \%$ CI 6-22) $v 0$ $(0.22-0.85) ; \mathrm{p}>0.2)$, with complete lumen obliteration at 8 weeks $(\mathrm{n}=4$, median patency $0.0(0-0) v 0.0(0-0)$, $\mathrm{p}>0.2$ ). In addition, $\mathrm{BALB} / \mathrm{c}$ allografts transplanted into the $\mathrm{C} 57 \mathrm{BL} / 6$ recipients under $\mathrm{C} 3 \mathrm{H}$ DST/anti-CD154 treatment were rejected, similar to $\mathrm{BALB} / \mathrm{C}$ allografts under anti-CD154 treatment alone $(\mathrm{n}=4, \mathrm{p}>0.2$ both at 6 and 8 weeks). Finally, $\mathrm{C} 3 \mathrm{H}$ allografts $(\mathrm{n}=5)$ transplanted at week 4 into $\mathrm{C} 57 \mathrm{BL} / 6$ mice that had already received a $\mathrm{BALB} / \mathrm{C}$ allograft at day 0 under cover of BALB/C DST/ anti-CD154 were fully rejected $(0.0(0.0) \%$ lumen patency) at 5 weeks, not significantly different $(p>0.2)$ from $\mathrm{C} 3 \mathrm{H}$ allografts $(\mathrm{n}=5)$ placed in C57BL6 animals without treatment, but significantly different $(p=0.01$, Wilcoxon rank sum test) from similarly treated C57BL6 animals that received a second BALB/C graft $(100(0.0) \%$ at 5 weeks).
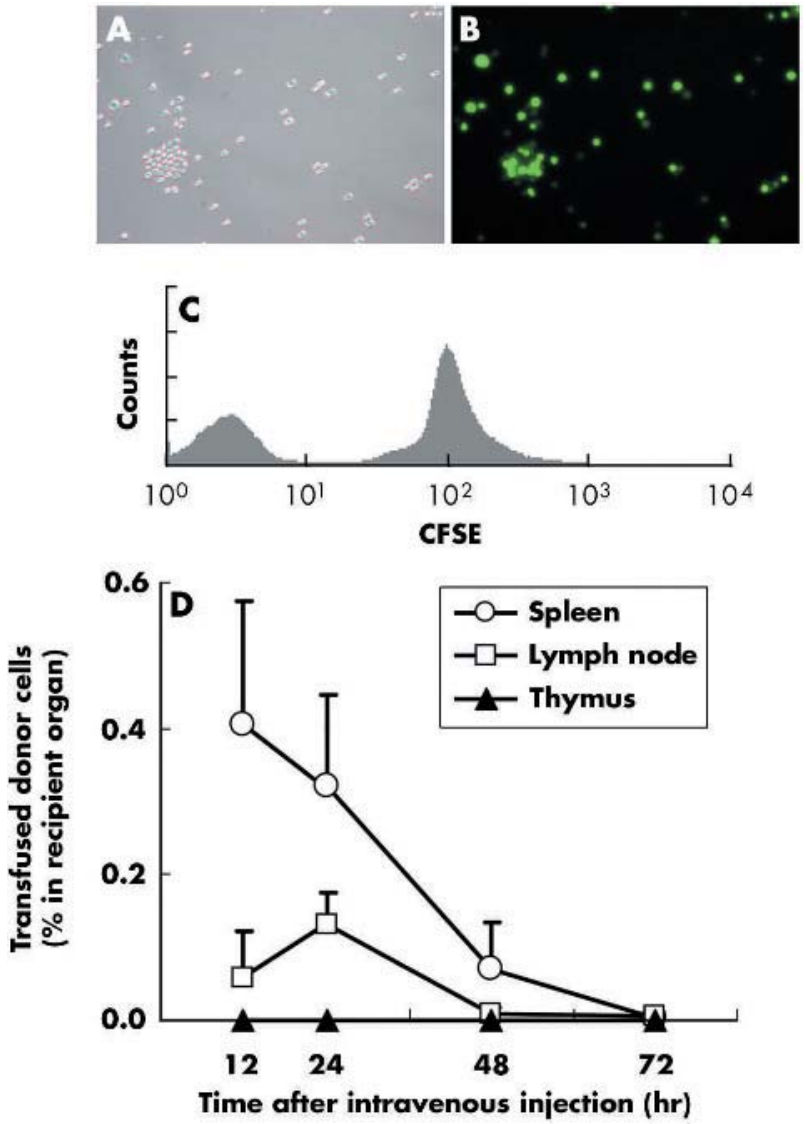

Figure 4 Flow cytometry shows that donor splenocytes home to secondary and primary lymphoid organs. Transfused donor cells were traced in vivo using CFSE labelling. Freshly isolated splenocytes readily acquired intracellular CFSE label (A, light microcopy; B fluorescence microscopy; C, flow cytometry). As an internal control, unlabelled splenocytes were mixed with labelled splenocytes (C). (D) A small number of transfused cells migrated to, and then rapidly disappeared from, the recipient lymphoid organs. Naïve C57BL/6 mice were transfused with $10^{7}$ freshly isolated CFSE labelled BALB/c splenocytes. The frequencies of CFSE positive cells within lymphoid organs (spleen, transplant draining lymph nodes, and thymus) were determined 12, 24, 48 and 72 hours after transfusion ( $n=4$ at each time point). The $y$ axis shows the percentages of cells within the recipient primary (thymus) and secondary (spleen and transplant draining lymph nodes) lymphoid organs. The fluorescence intensities of single cell suspensions from primary and secondary lymphoid organs were acquired by FACScan and analysed with FlowJo with a minimum of $10^{6}$ events for each analysis. Data are shown as mean (SD) percentages of CFSE positive cells within the whole respective lymphoid organs and are representative of three separate experiments.

\section{Kinetics of transfused donor splenocytes in recipient's secondary lymphoid organs}

In order to determine the fate and kinetics of transfused donor cells in vivo, donor (BALB/c) splenocytes were labelled with immunofluorescence dye before intravenous injection into naïve recipient (C57BL/6) mice (fig 4A-C). The frequencies of the transfused CFSE positive donor cells within the recipient's primary (thymus) and secondary (spleen and transplant draining (axillary) lymph nodes) lymphoid organs at 12, 24, 48 and 72 hours after transfusion are shown in fig 4D. At 24 hours after the transfusion, donor splenocytes were detected within the white pulp areas of the spleen $(\sim 0.35 \%$ of the resident splenocytes; figs $4 \mathrm{D}$ and $5 \mathrm{~A}-$ C) and, to a lesser extent, within the non-follicular areas of the axillary lymph nodes $(\sim 0.15 \%$ of the resident lymphocytes; figs $4 \mathrm{D}$ and 5D-F). These cells could not be detected 

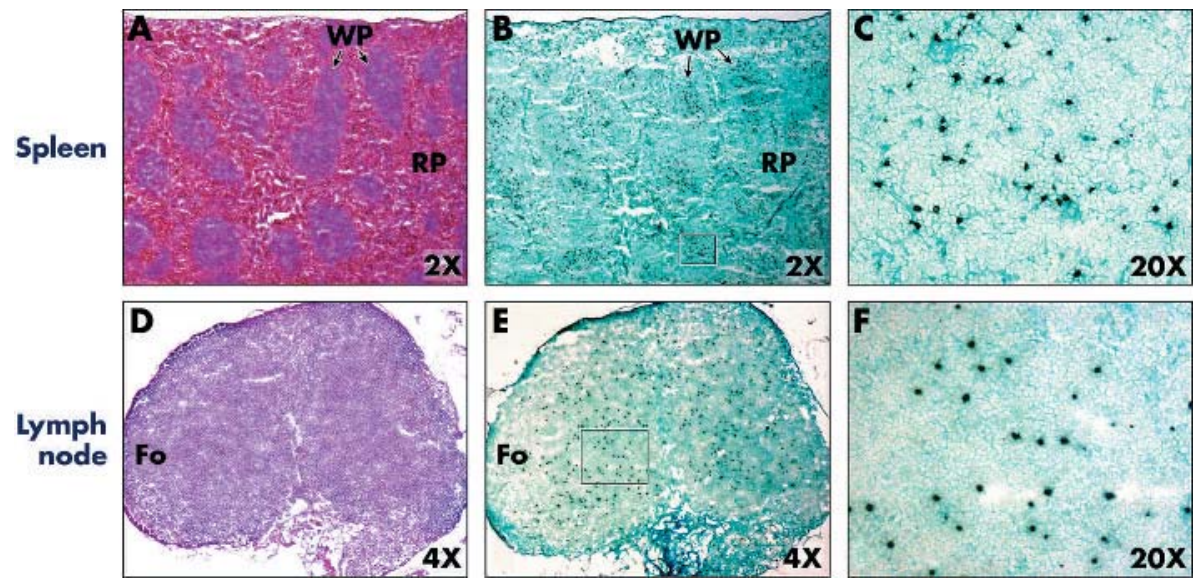

Figure 5 Representative histology primary and secondary lymphoid organs of recipient 12 hours after donor specific transfusion. Sections of spleen were examined by immunohistochemistry staining for CFSE label. The section was incubated with HRP conjugated rabbit anti-FITC antibody and the colour was developed with DAB. Transfused donor CFSE labelled T cells (dark brown) were localised primarily in the white pulp of the spleen (A-C) or non-follicular areas of the draining lymph nodes (D-F). Comparison H\&E stained sections are shown in A and D. WP, white pulp; RP, red pulp; Fo, follicular area.

within these lymphoid organs at 72 hours after transfusion (fig 4D). No significant donor cells were detected within the thymus at any time point $(<0.001 \%$, fig $4 \mathrm{D})$. When
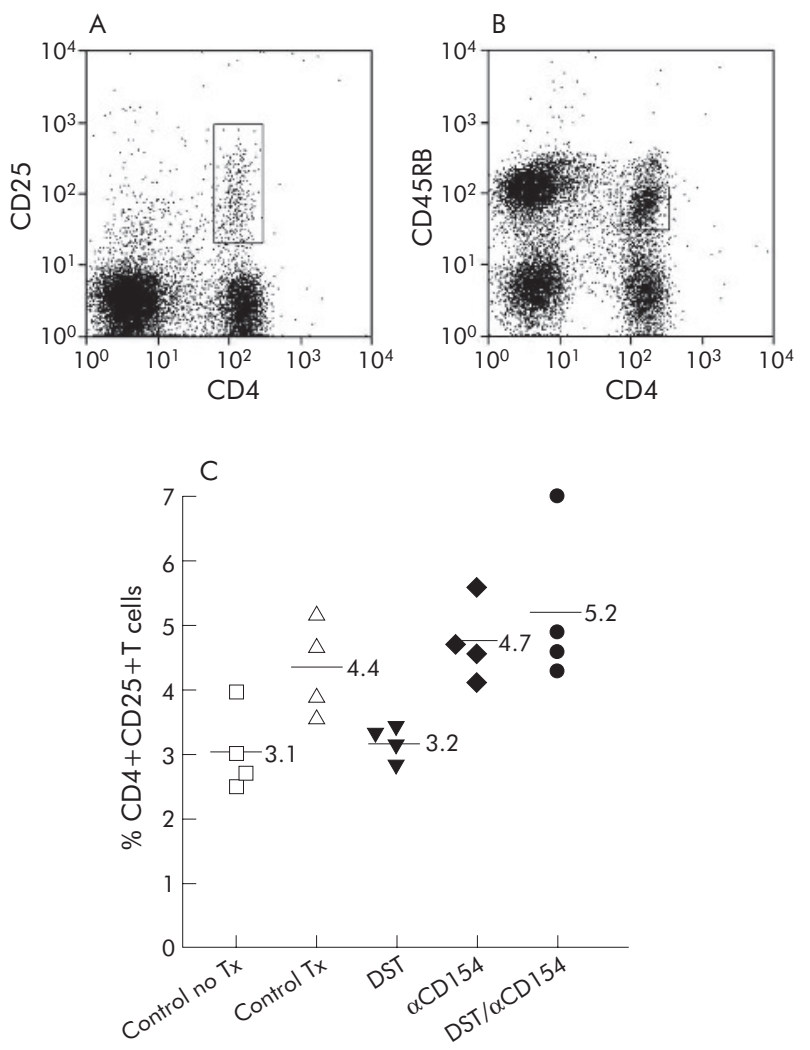

Figure 6 DST/anti-CD154 increased the percentage of CD4+CD25+ regulatory $T$ cells. Splenocytes were obtained from recipient mice 2 weeks after transplantation. Representative flow cytometric histograms of $C D 4+C D 25)$ and $C D 4+C D 45 R B^{\text {lo }}$ cells are shown in (A) and (B), respectively. CD4+ T cells were evaluated according to the presence of surface expression of CD25 or CD45RB marker (in boxes). A minimum of $10^{5}$ events was acquired for each analysis. (C) Mean percentage of CD4+CD25+ cells was significantly increased in the DST/anti-CD154 treatment group compared with the untreated transplant group ((5.2 $(1.3) \% \vee 4.4(0.8) \%, p=0.03)$. The results are representative of two separate experiments performed at 2 and 4 weeks after transplantation. Lymph node analyses gave similar results (data not shown).
anti-CD154 was co-administered, the kinetics of transfused donor splenocytes was not altered (data not shown).

\section{Effect of DST/anti-CD 154 protocol on frequencies of CD4+CD25+ or CD4+CD45RB ${ }^{\text {lo }}$ regulatory/suppressor T cells}

Using flow cytometry, CD4+ $\mathrm{T}$ cells derived from the recipient's secondary lymphoid organs at 2 and 4 weeks after transplantation were gated according to the surface expression of CD25 or CD45RB (fig 6A and B). The percentage of splenic CD4+CD25+ cells was significantly higher in the DST/anti-CD154 group than in the untreated transplant control group $(p=0.03)$, but only trended toward being higher compared with the group treated with antiCD154 alone $(p=0.19 ; 2$ week data shown in fig 6C). Similar results were found in both the CD4+CD25+ cells derived from recipient's draining lymph nodes and splenic CD4+CD45RB ${ }^{\text {lo }}$ cells at both 2 and 4 weeks (data not shown).

\section{DISCUSSION}

We report the long term survival of fully histo-incompatible airway allografts without the use of immunosuppressants in the mouse model of chronic lung rejection. This is one of the first reports where tolerance has been reliably achieved in any lung rejection model and, in addition, in an animal model of chronic graft rejection. Donor specific transplantation tolerance was successfully induced and maintained by a single treatment of donor splenocytes in combination with a short course of antagonistic non-T cell depleting anti-CD154 monoclonal antibody (DST/anti-CD154). By providing donor antigen specific signal while blocking the co-stimulation to the recipient's T cells within the secondary lymphoid organs, this combination protocol markedly suppressed the alloreactive $\mathrm{T}$ cell effector response and achieved donor specific transplantation tolerance. This protocol clearly induced transplantation tolerance via a peripheral mechanism(s) as it did not result in mixed chimerism or central deletion. In addition, we found that, during the induction phase of transplantation tolerance, the percentages of CD4+CD25+ or $\mathrm{CD} 4+\mathrm{CD} 45 \mathrm{RB}^{\text {lo }}$ regulatory/suppressor $\mathrm{T}$ cells within the spleen and draining lymph nodes of the recipient with the DST/anti-CD154 treatment were significantly increased compared with the no treatment group.

Alloimmunity is initiated by the recognition of alloantigen (allorecognition) present on the surface of donor (direct 
pathway) and/or recipient (indirect pathway) APCs to recipient $\mathrm{T}$ cells (signal 1 ). ${ }^{2}$ With the help of co-stimulatory signals (signal 2 ), allospecific $\mathrm{T}$ cells then become activated, proliferate, and differentiate into effector cells that, through powerful, complex, and well orchestrated immune cascades, ultimately destroy the graft. In this study, using the mouse heterotopic tracheal transplant model for chronic lung rejection, we evaluated the effectiveness of a combination DST/anti-CD154 protocol by exposing the recipient to donor antigens (signal 1) concomitant with co-stimulation (signal 2) blockade before transplantation. We found that the DST/ anti-CD154 protocol was very effective in inducing and maintaining airway allograft tolerance, in keeping with studies in other transplant mouse models showing improved islet, heart and skin allograft survival in euthymic and thymectomised recipients. ${ }^{9-11}$ In lung transplantation, several studies have reported the prolongation of murine heterotopic tracheal allograft survival without the use of immunosuppressants. ${ }^{21-25}$ However, those studies found significant improvement of end points of allograft survival at no more than 42 days after the last treatment. In addition, when lymphocyte proliferation (MLR) was reported, the treatment did not result in donor specific suppression. ${ }^{22}{ }^{23}$ In sharp contrast, we have shown that the DST/anti-CD154 protocol maintained nearly complete airway patency beyond 100 days after the final anti-CD154 administration, indicating that the DST/anti-CD154 protocol is by far the most effective protocol to date for inducing tolerance in murine models of chronic lung rejection. We also showed that this state of transplantation tolerance was donor specific since the (BALB/C) DST/ anti-CD154 did not prolong survival of the fully histoincompatible third party $(\mathrm{C} 3 \mathrm{H})$ grafts nor did the third party $(\mathrm{C} 3 \mathrm{H})$ DST/anti-CD154 prolong survival of BALB/C allografts in the $\mathrm{C} 57 \mathrm{BL} / 6$ recipients, and nor could third party $(\mathrm{C} 3 \mathrm{H})$ grafts be successfully engrafted into C57BL/6 mice who had received a BALB/c graft under cover of (BALB/C) DST/ antiCD154. Donor specific signal provided by the DST/antiCD154 protocol may selectively capture allospecific $\mathrm{T}$ cell repertoires and be responsible for this distinction.

The mechanism(s) by which the DST/anti-CD154 protocol induces tolerance has not been fully elucidated but has been studied in non-lung transplantation models. DST/anti-CD154 induces Th2 polarisation ${ }^{10}$ and the deletion of the alloreactive CD8+ cells. ${ }^{26}$ These effects appear to be CTLA4 dependent ${ }^{11}$ and require the presence of a subset of CD4+ $\mathrm{T}$ cells, presumably regulatory/suppressor cells. ${ }^{11}{ }^{27}$ In this study we have shown, firstly, that each component was mandatory since treatment with DST alone or anti-CD154 alone was far less effective than the combination. This finding is consistent with previous findings showing that donor whole blood transfusion modestly prolonged the longevity of human renal allografts ${ }^{28}$ and that anti-CD154 used as monotherapy did not induce mouse cardiac allograft tolerance ${ }^{29}$ due to its inability to block rejection elicited by CD8+ or CD4+ effector T cells. Secondly, while it remains controversial, ${ }^{30}$ it is generally believed that antigen recognition occurs in the secondary lymphoid organs. ${ }^{31}{ }^{32}$ In this study we showed that DST cells homed to the recipient's secondary lymphoid organs, primarily to the white pulp of the spleen or non-follicular areas of the lymph nodes, respectively, extending a previous observation in the spleen after allogeneic B cell transfusion. ${ }^{33}$ In addition, the rapid disappearance of transfused donor cells from these lymphoid organs (within 72 hours) in the presence or absence of anti-CD154 suggests that mixed chimerism was not achieved, corroborating and extending the findings of others. ${ }^{18} 26$ Thirdly, transfused donor cells were undetectable in the recipient's thymi at any time during the study, suggesting that central (intrathymic) deletion is less likely to account for tolerance induction by this protocol.
Finally, varying numbers of lymphocytes infiltrated the tolerised allografts but did not lead to tissue rejection, suggesting either non-specific inflammation (that is, $\mathrm{T}$ cells without donor specificities) or the recruitment of anergic $\mathrm{T}$ cells. Taken together, our data are in agreement with the hypothesis that the DST/anti-CD154 protocol induces transplantation tolerance by a peripheral mechanism(s) leading to a suppressed donor specific CTL response at an effector function level and, although not studied here, the possibility of generating anergic $\mathrm{T}$ cells that traffic to the graft.

Regulatory $\mathrm{T}\left(\mathrm{T}_{\mathrm{R}}\right)$ cells, including naturally arising $\mathrm{T}_{\mathrm{R}}$ cells $\left(\mathrm{CD} 4+\mathrm{CD} 25+\right.$ or $\left.\mathrm{CD} 4+\mathrm{CD} 45 \mathrm{RB}^{\mathrm{lo}}\right)$ have been evident with many tolerance inducing protocols in many transplant and autoimmunity models and may be responsible for tolerance induction. While others have suggested that, under the DST/ anti-CD154 protocol, the presence of a subset of CD4+ cells is critical for prolonged allograft survival, ${ }^{11} 182734$ they have not identified this subset of CD4+ cells. In this study we found a significant increase in the percentage of CD4+CD25+ and CD4+CD45RB ${ }^{\text {lo }} \mathrm{T}_{\mathrm{R}}$ cells within the spleen and lymph nodes of recipient animals treated with this protocol at 2 and 3 weeks after transplantation, suggesting a role for these cells in tolerance induction. However, since the functions of these cells were not evaluated in vitro or in vivo and we did not measure FoxP3 expression, we cannot prove that these cells were responsible for tolerance induction. The precise mechanisms by which the DST/anti-CD154 protocol induces and maintains lung transplant tolerance, including the involvement of $T_{R}$ cells, have yet to be elucidated and are under current investigation in our laboratory.

This study has several potential limitations. Firstly, the mouse model of chronic lung rejection has limitations as previously described ${ }^{14-17}$ but it remains one of the best (if not the best) model for chronic rejection since orthotopic models do not characteristically reproduce human OB. ${ }^{35} 36$ Nonetheless, this is the first study to demonstrate the use of this model of chronic lung rejection and tolerance induction protocol to accomplish interventional lung transplantation tolerance. The merits of this model therefore argue for further testing of lung transplant tolerance inducing protocols using this and other models, including those that primarily model acute rejection (such as the rat orthotopic model). Secondly, the brevity and simplicity of the DST/antiCD154 protocol obviates the need for non-specific immunosuppression. However, in an effort to facilitate pre-emptive tolerance induction of the allospecific $\mathrm{T}$ cell compartment, DST/anti-CD154 must be introduced before transplantation. Application of this protocol would therefore be restricted from the cadaveric donor transplant setting in clinical medicine. Evaluation of the effectiveness of this type of protocol-when DST is given at the time of or a few hours before transplantation (that is, at -1 to -6 hours)-are being investigated currently with the goal of improving its clinical application. Thirdly, although the operational criteria defining indefinite graft tolerance is graft survival for more than 100 days, tracheal allografts under the DST/anti-CD154 protocol may not truly maintain indefinite tracheal graft survival as they displayed a gradual progressive loss of differentiated epithelium over time and completely lost epithelialisation between 14 and 16 weeks. The loss of the epithelial lining of the murine tracheal allograft lumen has been consistently observed preceding lumen obliteration, ${ }^{12-15} 3738$ suggesting that these grafts may be rejected eventually. The potential benefits of additional DST and/or anti-CD154 treatment(s) used at later time points after transplantation to suppress new allospecific thymic emigrants remain to be determined.

In conclusion, long term donor specific airway allograft tolerance in the stringent, fully histo-incompatible, heterotopic 
tracheal transplant model of chronic lung rejection can be induced and maintained by the combination treatment of a single donor specific transfusion and a short course of the monoclonal anti-CD154 antibody around the time of transplantation. This finding has brought us a significant step closer to successful tolerance induction in lung transplantation. Further studies are needed to identify the mechanisms by which this protocol achieves tolerance and to modify this protocol to become more clinically applicable, thereby positioning clinical lung transplant tolerance trials into situations currently occupied only by other organ transplants.

\section{Authors' affiliations}

W Chalermskulrat, I P Neuringer, R C Park, D G Sterka, P McNeillie, R M Aris, Division of Pulmonary Diseases and Critical Care Medicine and the Lung Transplantation Program, University of North Carolina, School of Medicine, Chapel Hill, NC, USA

K P McKinnon, W J Brickey, B R Long, J P Ting, Department of Microbiology and Immunology, University of North Carolina, School of Medicine, Chapel Hill, NC, USA

K P McKinnon, W J Brickey, J P Ting, Lineberger Comprehensive Cancer Center, University of North Carolina, School of Medicine, Chapel Hill, NC, USA

R J Noelle, Department of Microbiology and Immunology, Dartmouth Medical School, Lebanon, NH, USA

This research was supported by the American Lung Association (ALA) (WC and IPN), the Cystic Fibrosis Foundation (RMA and WC), and the National Institute of Health HL67178 (IPN), Al29564 (JPT) and DK38108 (JPT).

Competing interests: none declared.

\section{REFERENCES}

1 Massicot-Fisher J, Noel P, Madsen JC. Recommendations of the National Heart, Lung and Blood Institute Heart and Lung Tolerance Working Group. Transplantation $2001 ; 72: 1467-70$.

2 Wekerle T, Kurtz J, Bigenzahn S, et al. Mechanisms of transplant tolerance induction using costimulatory blockade. Curr Opin Immunol 2002; 14:592-600.

3 Frauwirth KA, Thompson CB. Activation and inhibition of lymphocytes by costimulation. J Clin Invest 2002;109:295-9.

4 Lenschow DJ, Zeng Y, Thistlethwaite JR, et al. Long-term survival of xenogeneic pancreatic islet grafts induced by CTLA4lg. Science 1992;257:789-92.

5 Baliga P, Chavin KD, Qin L, et al. CTLA4lg prolongs allograft survival while suppressing cell-mediated immunity. Transplantation 1994;58:1082-90.

6 Onodera K, Chandraker A, Volk HD, et al. Distinct tolerance pathways in sensitized allograft recipients after selective blockade of activation signal 1 or signal 2. Transplantation 1999;68:288-93.

7 Quezada SA, Jarvinen L, Lind EF, et al. CD40/CD154 interactions at the interface of tolerance and immunity. Annu Rev Immunol 2004;22:307-28.

8 Rumbley CA, Silver SJ, Phillips SM. Dependence of murine obstructive airway disease on CD40 ligand. Transplantation 2001;72:1616-25.

9 Parker DC, Greiner DL, Phillips NE, et al. Survival of mouse pancreatic islet allografts in recipients treated with allogeneic small lymphocytes and antibody to CD40 ligand. Proc Natl Acad Sci USA 1995;92:9560-4.

10 Hancock WW, Sayegh MH, Zheng XG, et al. Costimulatory function and expression of CD40 ligand, CD80, and CD86 in vascularized murine cardiac allograft rejection. Proc Natl Acad Sci USA 1996;93:13967-72.

11 Markees TG, Phillips NE, Noelle RJ, et al. Prolonged survival of mouse skin allografts in recipients treated with donor splenocytes and antibody to CD40 ligand. Transplantation 1997;64:329-35.

12 Hele DJ, Yacoub MH, Belvisi MG. The heterotopic tracheal allograft as an animal model of obliterative bronchiolitis. Respir Res 2001;2:169-83.

13 Neuringer IP, Charlermskulrat W, Aris RM. Obliterative bronchiolitis: basic mechanisms of chronic lung rejection. J Heart Lung Transplant 2005;24:3-19.
14 Neuringer IP, Mannon RB, Coffman TM, et al. Immune cells in a mouse airway model of obliterative bronchiolitis. Am J Respir Cell Mol Biol 1998; 19:379-86.

15 Chalermskulrat W, Neuringer IP, Brickey WJ, et al. Hierarchical contributions of allorecognition pathways in chronic lung rejection. Am J Respir Crit Care Med 2003; 167:999-1007.

16 Neuringer IP, Aris RM, Burns KA, et al. Epithelial kinetics in mouse heterotopic tracheal allografts. Am J Transplant 2002;2:410-9.

17 Aris RM, Walsh S, Chalermskulrat W, et al. Growth factor upregulation during obliterative bronchiolitis in the mouse model. Am J Respir Crit Care Med 2002;166:417-22.

18 Markees TG, Phillips NE, Gordon EJ, et al. Long-term survival of skin allografts induced by donor splenocytes and anti-CD154 antibody in thymectomized mice requires CD4(+) T cells, interferon-gamma, and CTLA4. $J$ Clin Invest 1998;101:2446-55.

19 Shirasugi N, Adams AB, Durham MM, et al. Prevention of chronic rejection in murine cardiac allografts: a comparison of chimerism- and nonchimerisminducing costimulation blockade-based tolerance induction regimens. $\mathrm{J}$ Immunol 2002;169:2677-84.

20 Felix NJ, Brickey WJ, Griffiths R, et al. H2-DMalpha(-/-) mice show the importance of major histocompatibility complex-bound peptide in cardiac allograft rejection. J Exp Med 2000;192:31-40.

21 Morikawa M, Brazelton TR, Berry GJ, et al. Prolonged inhibition of obliterative airway disease in murine tracheal allografts by brief treatment with anti-leukocyte function-associated antigen-1 (CD1 la) monoclonal antibody. Transplantation 2001;71:1616-21.

22 Yamada A, Konishi K, Cruz GL, et al. Blocking the CD28-B7 T-cell costimulatory pathway abrogates the development of obliterative bronchiolitis in a murine heterotopic airway model. Transplantation 2000:69:743-9.

23 Konishi K, Inobe M, Yamada A, et al. Combination treatment with FTY720 and CTLA4lgG preserves the respiratory epithelium and prevents obliterative disease in a murine airway model. J Heart Lung Transplant 2002;21:692-700.

24 Leonard CT, Soccal PM, Berry GJ, et al. PG490-88, a derivative of triptolide, attenuates obliterative airway disease in a mouse heterotopic tracheal allograft model. J Heart Lung Transplant 2002;21:1314-8.

25 Duncan SR, Capetanakis NG, Lawson BR, et al. Thymic dendritic cells traffic to thymi of allogeneic recipients and prolong graft survival. J Clin Invest 2002; 109:755-64.

26 Niimi M, Pearson TC, Larsen CP, et al. The role of the CD40 pathway in alloantigen-induced hyporesponsiveness in vivo. J Immunol 1998;161:5331-7.

27 van Maurik A, Herber M, Wood KJ, et al. Cutting edge: CD4(+)CD25(+) alloantigen-specific immunoregulatory cells that can prevent CD8(+) T cellmediated graft rejection: implications for anti-CD154 immunotherapy. $J$ Immunol 2002; 169:5401-4.

28 Brennan DC, Mohanakumar T, Flye MW. Donor-specific transfusion and donor bone marrow infusion in renal transplantation tolerance: a review of efficacy and mechanisms. Am J Kidney Dis 1995;26:701-15

29 Jones ND, Van Maurik A, Hara M, et al. CD40-CD40 ligand-independent activation of CD8+ T cells can trigger allograft rejection. J Immunol 2000;165:1111-8.

30 Kreisel D, Krupnick AS, Gelman AE, et al. Non-hematopoietic allograft cells directly activate CD8+T cells and trigger acute rejection: an alternative mechanism of allorecognition. Nat Med 2002;8:233-9.

31 Mondino A, Khoruts A, Jenkins MK. The anatomy of T-cell activation and tolerance. Proc Natl Acad Sci USA 1996:93:2245-52.

32 Lakkis FG, Arakelov A, Konieczny BT, et al. Immunologic 'ignorance' of vascularized organ transplants in the absence of secondary lymphoid tissue. Nat Med 2000;6:686-8.

33 lyoda T, Shimoyama S, Liu K, et al. The CD8+ dendritic cell subset selectively endocytoses dying cells in culture and in vivo. J Exp Med 2002; 195: 1289-302.

34 Wood KJ, Sakaguchi S. Regulatory lymphocytes: regulatory T cells in transplantation tolerance. Nat Rev Immunol 2003;3:199-210.

35 Kondo T, Marchevsky AM, Prehn J, et al. Evidence of complete tolerance in a model of rat lung allografts. Transplantation 1991;52:928-31.

36 Matsumura Y, Marchevsky A, Zuo XJ, et al. Assessment of pathological changes associated with chronic allograft rejection and tolerance in two experimental models of rat lung transplantation. Transplantation 1995:59:1509-17.

37 Boehler A, Chamberlain D, Kesten S, et al. Lymphocytic airway infiltration as a precursor to fibrous obliteration in a rat model of bronchiolitis obliterans. Transplantation 1997;64:311-7.

38 Hertz MI, Jessurun J, King MB, et al. Reproduction of the obliterative bronchiolitis lesion after heterotopic transplantation of mouse airways. Am J Pathol 1993;142:1945-51. 